\title{
Corrosión atmosférica de metales en condiciones climáticas extremas
}

\author{
B. CHICO, D. DE LA FUENTE Y M. MORCILLO \\ Dept. de Ingeniería de Materiales, Degradación y Durabilidad; Centro Nacional de \\ Investigaciones Metalúrgicas (CENIM), CSIC; Avda. Gregorio del Amo, 8 28040-Madrid
}

\begin{abstract}
En el presente trabajo se realiza un análisis de la influencia de condiciones climáticas extremas en la corrosión atmosférica de metales. Este fenómeno de corrosión está determinado, principalmente, por el tiempo durante el cual permanece humedecida la superficie metálica (tiempo de humectación), presencia de contaminantes atmosféricos y la temperatura ambiente. Se analizan distintos comportamientos observados en metales expuestos a unas condiciones atmosféricas extremas de temperatura y humedad relativa, en presencia o no de contaminantes atmosféricos. Los resultados que se exponen fueron obtenidos en dos programas internacionales sobre corrosión atmosférica: Programa ISOCORRAG, de la International Standards Organization (ISO), y el Proyecto Iberoamericano MICAT (Mapa Iberoamericano de Corrosividad Atmosférica), del Programa Iberoamericano de Ciencia y Tecnología para el Desarrollo (CYTED).
\end{abstract}

Palabras clave: Corrosión atmosférica, Meteorología

\section{Atmospheric corrosion of metals in extreme climatic conditions}

This paper analyses the influence of extreme climatic conditions on the atmospheric corrosion of metals. This corrosion phenomenon is determined mainly by the time that moisture is retained on the metallic surface (time of wetness), the presence of atmospheric pollutants, and the air temperature. An analysis is made of different behaviours observed in metals exposed in atmospheric conditions of extreme temperature and relative humidity, in the presence and absence of atmospheric pollutants. The results presented here were obtained in two international research programmes on atmospheric corrosion: ISOCORRAG programme, of the International Standards Organization (ISO), and the Ibero-American MICAT project (Ibero-American Map of Atmospheric Corrosivity), of the Ibero-American Science and Technology for Development Programme (CYTED).

Keywords: Atmospheric corrosion, Meteorology

\section{INTRODUCCION}

Es conocido que el proceso de corrosión de metales expuestos a la atmósfera es de tipo electroquímico. Este proceso de corrosión tiene lugar cuando la superficie se encuentra humedecida debido a diferentes fenómenos meteorológicos como la lluvia, condensación de humedad (rocío), niebla, etc. El tiempo durante el cual permanece humedecida la superficie metálica, conocido como tiempo de humectación (TDH), es, por tanto, un factor determinante de la velocidad de corrosión atmosférica. Un indicador frecuentemente utilizado para estimar el TDH es calcular el tiempo durante el cual se cumplen simultáneamente las condiciones de humedad relativa $(\mathrm{HR})>80 \%$ y temperatura $(\mathrm{T})>$ $0^{\circ} \mathrm{C}(1)$.

Por tanto, de entre los factores climáticos, la HR y T influyen de una manera decisiva en la magnitud del fenómeno de corrosión atmosférica, existiendo en la literatura diversos estudios que tratan este tema $(2,3)$. También existen estudios acerca de la influencia de otras variables climáticas, como dirección y velocidad del viento en el lugar de exposición $(4,5)$, etc.

Por otro lado, la presencia de contaminantes atmosféricos incrementa considerablemente la velocidad de corrosión. El $\mathrm{NaCl}$ y el $\mathrm{SO}_{2}$, son los agentes corrosivos más comunes de la atmósfera, encontrándose en la bibliografía diversos trabajos que relacionan velocidades de corrosión y salinidad atmosférica (6) y dióxido de azufre $(7,8)$.

La gran diversidad de condiciones meteorológicas y de conta-
TABla I. CORROSIÓN ANUAL DEL ACERO AL CARBONO, ZINC, COBRE Y ALUMINIO EN ATMÓSFERAS DE LA REGIÓN IBEROAMERICANA (9).

\begin{tabular}{|c|c|c|c|c|c|c|}
\hline $\begin{array}{l}\text { Tipo de } \\
\text { atmósfera }\end{array}$ & Lugar & País & $\begin{array}{c}\text { Acero al } \\
\text { carbono } \\
(\mu \mathrm{m})\end{array}$ & $\begin{array}{l}\text { Zinc } \\
(\mu \mathrm{m})\end{array}$ & $\begin{array}{l}\text { Cobre } \\
(\mu \mathrm{m})\end{array}$ & $\begin{array}{l}\text { Aluminio } \\
\left(\mathrm{g} \cdot \mathrm{m}^{-2}\right)\end{array}$ \\
\hline \multirow{4}{*}{ Rural } & Cuzco & Perú & 1,4 (mín) & 0,40 & 0,09 (mín) & 0,02 (mín.) \\
\hline & S. Juan & Argentina & 4,9 & 0,21 & 0,17 & 0,08 \\
\hline & Granada & España & 8,5 & 0,11 (mín) & 0,22 & 0,04 \\
\hline & Cotové & Colombia & 19.6 & 0,33 & 0.73 & 0,14 \\
\hline \multirow{2}{*}{ Urbana } & México & México & 9,7 & 0,82 & 0,64 & 0,12 \\
\hline & Sao Paulo & Brasil & 20,6 & 1,21 & 1.69 & 0,56 \\
\hline \multirow{2}{*}{ Marina } & A. do Cabo & Brasil & 165,4 & 4,87 & 4,12 & 1,68 \\
\hline & Ubatuba & Brasil & 302,0 & 7.07 & 3.29 & 1.17 \\
\hline Industrial & Petrox & Chile & 167,2 & 8.58 (máx) & 5,35 (máx.) & 5.43 (máx.) \\
\hline Urbana-Marina & Sines & Portugal & 365,0 (máx) & 4,03 & 3,61 & 3,79 \\
\hline \multirow{2}{*}{ Industrial-Marina } & Leixoes & Portugal & 72.4 & 2.51 & 4.32 & 4,09 \\
\hline & & $\mathrm{R}=\mathrm{máx} . / \mathrm{mín}$ & 261 & 78 & 59 & 272 \\
\hline
\end{tabular}

minación origina que las velocidades de corrosión de metales expuestos a la atmósfera sean decenas e incluso centenares de veces mayores en unos lugares que en otros. En la tabla I se exponen los rangos de velocidades de corrosión encontrados para el acero al carbono, zinc, cobre y aluminio en una red Iberoamericana de ensayo de materiales en la atmósfera (Proyecto MICAT) (9). Mediante un tratamiento estadístico del conjunto de datos se pueden obtener ecuaciones de correlación que hacen posible predecir la velocidad de corrosión a partir de los parámetros meteorológicos y de la contaminación. Las fun- 
ciones de daño obtenidas para diferentes metales en el Proyecto MICAT (9) fueron:

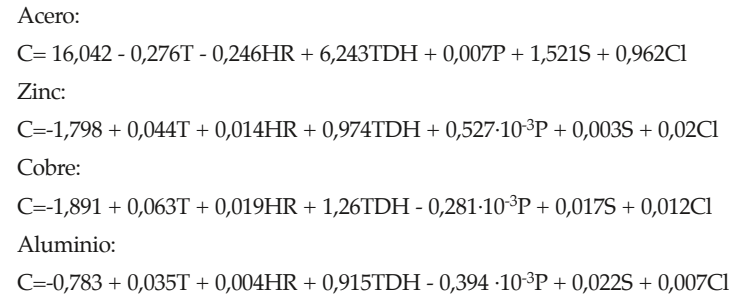

donde C: corrosión anual, en $\mu \mathrm{m}$ (aluminio en $\mathrm{g} / \mathrm{m}^{2}$ ); HR: humedad relativa media anual, en \%; T: temperatura media anual, en ${ }^{\circ} \mathrm{C}$; P: volumen de precipitación, en mm/año; TDH: fracción anual del número de horas / año en que la $\mathrm{HR}>80 \%$ y $\mathrm{T}>0^{\circ} \mathrm{C}$; $\mathrm{S}$ : contaminación por $\mathrm{SO}_{2}$, en $\mathrm{mg} \mathrm{SO} / \mathrm{m}^{2}$.d y Cl: contaminación por cloruros, en $\mathrm{mg} \mathrm{Cl}^{-} / \mathrm{m}^{2}$.d.

En el presente trabajo se analiza el comportamiento de metales expuestos a la atmósfera en condiciones extremas de temperatura y humedad relativa, en presencia o no de contaminantes atmosféricos, a partir de los resultados obtenidos en dos programas internacionales de investigación sobre corrosión atmosférica: Programa ISOCORRAG (10) y el Proyecto Iberoamericano MICAT (9)

\section{CORROSION ATMOSFERICA DE METALES EXPUESTOS EN CONDICIONES EXTREMAS DE TEMPERATURA Y HUMEDAD RELATIVA}

\subsection{Atmósferas puras, sin contaminación por cloruros ni} dióxido de azufre

En las figuras 1 y 2 se muestran valores anuales de corrosión en función de la humedad relativa (datos base MICAT) y temperatura (datos base MICAT e ISOCORRAG), respectivamente. En las mismas están consideradas atmósferas de tipo rural, aparentemente no contaminadas por cloruros ni dióxido de azufre.

En la figura 1 se observa una relación polinómica entre las variables humedad relativa y velocidad de corrosión del zinc. A humedades relativas extremadamente bajas (Arequipa, Perú, con $\mathrm{HR}=35 \%, \mathrm{~T}=16,8^{\circ} \mathrm{C}$ ), la velocidad de corrosión del zinc es $0,23 \mu \mathrm{m} / \mathrm{a}$, mientras que a humedades altas y temperaturas ligeramente inferiores (San Pedro, Colombia, con $\mathrm{HR}=90 \%$ y T $=11,5^{\circ} \mathrm{C}$ ) la velocidad se incrementa a $3,29 \mu \mathrm{m} / \mathrm{a}$.

En la figura 2, donde se representan velocidades de corrosión del acero en función de la temperatura, cabe destacar el valor de corrosión extremadamente bajo que se obtiene en la estación de Ojmajkon. En la tabla II se muestran, para este lugar de exposición, los valores de corrosión obtenidos para el zinc y cobre, que al igual que para el acero, son extremadamente bajos. La estación de Ojmajkon está situada en el interior de Siberia Oriental. Esta región se caracteriza por un clima extremadamente continental con fuertes contrastes térmicos (inviernos muy largos y rigurosos con temperaturas medias de hasta $-40^{\circ} \mathrm{C}$ ) y de precipitación. Las bajas temperaturas reinantes en Ojmajkon originan que la superficie metálica esté cubierta de una capa de hielo durante tiempos prolongados a lo largo del año, impidiendo considerablemente el desarrollo del proceso de corrosión.

El clima polar, como el continental, también se caracteriza por fuertes contrastes térmicos. Las temperaturas medias anuales son extremadamente bajas, superando $\operatorname{los} 0^{\circ} \mathrm{C}$ en pocos meses, 3 ó 4, y las precipitaciones son escasas. Estas atmósferas son especiales desde el punto de vista de la corrosión metálica ya
TABla II. CORROSIÓN ANUAL (MEDIA 6 AÑOS DE EXPOSICIÓN) DEL ACERO, ZINC Y COBRE, Y TEMPERATURA MEDIA, OBTENIDOS EN OJMAJKON (RUSIA).

\begin{tabular}{|c|c|c|c|c|}
\hline \multirow{2}{*}{$\begin{array}{c}\text { Lugar de } \\
\text { exposición }\end{array}$} & \multirow{2}{*}{,${ }^{\circ} \mathrm{C}$} & \multicolumn{3}{|c|}{ Velocidad de corrosión, $\mu \mathrm{m} / \mathrm{año}$} \\
\cline { 3 - 5 } & & Acero & Zinc & Cobre \\
\hline Ojmajkon & -15 & 0,8 & 0,36 & 0,08 \\
\hline
\end{tabular}

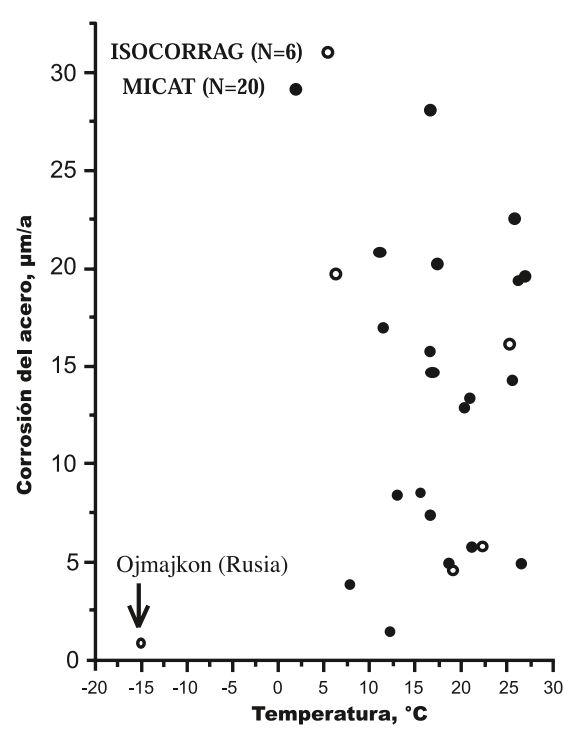

Fig. 2. Relación entre temperatura y velocidad de corrosión del acero expuesto a diferentes atmósferas en ausencia de contaminantes.

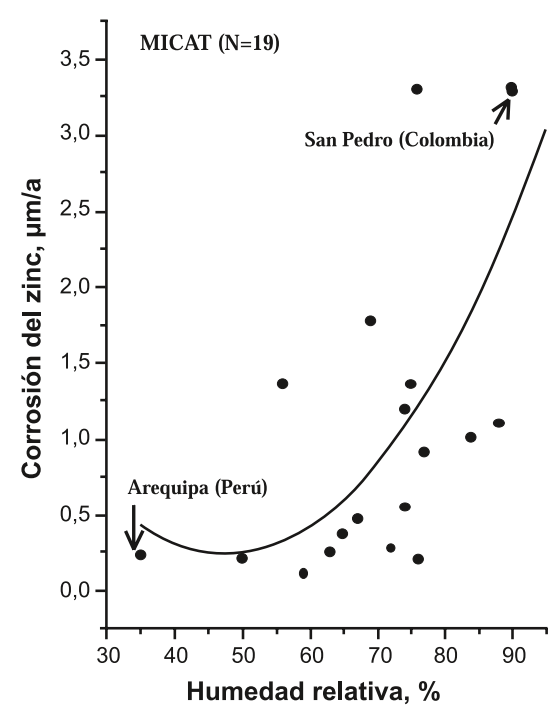

Fig. 1. Relación entre humedad relativa y velocidad de corrosión del zinc expuesto a diferentes atmósferas en ausencia de contaminantes.

que la superficie del metal se encuentra cubierta la mayor parte del tiempo de una capa de hielo de espesor variable. Esta capa de hielo reduce el acceso del oxígeno a la superficie metálica y el tiempo de humectación de ésta, disminuyendo las velocidades de corrosión hasta valores extremadamente bajos. En la tabla III se muestran datos obtenidos de la bibliografía (11-13) de metales expuestos a atmósferas con clima polar. La velocidad de corrosión más baja se ha medido en la estación de Vostok, en la Antártida, donde se ha registrado la temperatura más fría de la tierra $\left(-89,6^{\circ} \mathrm{C}\right.$ en julio de 1983$)$.

Sin embargo, estos valores de corrosión tan extremadamente bajos, no son exclusivos de climas caracterizados por temperaturas muy bajas, como lo demuestran las velocidades de corro- 
sión medidas en Cuzco (Perú) (15). En la tabla IV se muestran datos de corrosión anuales para el acero al carbono y variables meteorológicas y de contaminación, de probetas expuestas a la atmósfera rural de Cuzco. Esta atmósfera se aparta de las anteriores, exhibiendo períodos de humectación anual aproximadamente del $32 \%$ y precipitaciones del orden de $650 \mathrm{~mm} /$ año, condiciones que en principio no deberían impedir de manera acusada el proceso de corrosión atmosférica. Sin que se tenga una explicación del todo satisfactoria, parece ser que la atmósfera de Cuzco es extremadamente pura, limitandose la actividad electroquímica sobre la superficie metálica (15).

Climas caracterizados por temperaturas medias elevadas, bajas humedades relativas y precipitaciones muy escasas, es decir, un régimen climático de tipo desértico, los tiempos de humectación de la superficie metálica son muy cortos, dando origen a unas velocidades de corrosión extremadamente bajas. Así, por ejemplo, en Phoenix (Arizona, USA) (11), se han recogido datos de 4,6 $\mu \mathrm{m}$ / año para el acero al carbono. Datos meteorológicos medidos en Phoenix en los años 1961-90 (16) dan una temperatura media de $22,6^{\circ} \mathrm{C}$ (con un máximo de $41,1^{\circ} \mathrm{C}$ registrado en el mes de julio), una humedad relativa media de $37 \%$ y una precipitación media de $194 \mathrm{~mm}$.

En la figura 2 se presenta la variación de la corrosión del acero con la temperatura media anual en un conjunto de condiciones atmosféricas. No se infiere un papel determinante de la temperatura en la corrosión atmosférica de este metal, controlado quizás por otras variables meteorológicas: $\mathrm{HR}$, pluviosidad, etc.

\subsection{Atmósferas contaminadas por cloruros procedentes del mar}

En la tabla V se muestran valores de corrosión de probetas expuestas en el Artico y en la Antártida, a pocos metros de distancia al mar. Se obtienen valores más altos de lo esperado, teniendo en cuenta los cortos tiempos de humectación en este tipo de climas.

Dentro del Proyecto MICAT (9) se consideraron dos estaciones antárticas, Jubany (Argentina) y Artigas (Uruguay). En la tabla VI se exponen los datos anuales (media tres años de exposición) de variables medioambientales y de contaminación, y velocidades de corrosión para el acero al carbono, zinc, cobre y aluminio. Las velocidades obtenidas son menores de lo que se esperaría para atmósferas marinas de idéntica contaminación por cloruros pero de temperaturas medias más altas (9), a excepción del aluminio. La corrosión de este metal alcanza cotas más elevadas que en otras atmósferas marinas.

Los productos de corrosión formados sobre los diferentes metales se caracterizaron morfológicamente utilizando un equipo SEM/EDS (9) y químicamente mediante técnicas XRD, FTIR y espectroscopía Mössbauer (9). En las figuras 3 y 4 se muestran micrografías de secciones transversales de acero y zinc, respectivamente, expuestas a la atmósfera de Jubany, donde se observa la formación de profundas picaduras. Estas picaduras pueden formarse debido a la acción de una solución de elevada concentración de cloruros retenida bajo la capa de hielo formada sobre la superficie metálica. Diversos investigadores $(17,18)$ han demostrado que debajo de capas de hielo es posible la actividad de corrosión electroquímica.

En la figura 5 y 6 se exponen micrografías de la superficie del cobre y del aluminio, respectivamente, expuestas a la atmósfera de Jubany. En ellas es observable el agrietamiento de las películas de corrosión debido a la deshidratación durante los ciclos de secado de los cloruros básicos hidratados de tipo gel, que se forman sobre la superficie metálica.
TABLA III. VELOCIDADES DE CORROSIÓN OBTENIDAS EN DIFERENTES ESTACIONES SITUADAS EN EL INTERIOR DE LAS ZONAS ARTICA Y ANTÁRTICA.

\begin{tabular}{|c|c|c|c|}
\cline { 2 - 4 } \multicolumn{1}{c|}{} & Lugar de exposición & $\begin{array}{c}\text { Distancia } \\
\text { al mar, } \\
\text { Km }\end{array}$ & $\begin{array}{c}\text { Velocidad de } \\
\text { corrosión del acero, } \\
(\mu \mathrm{m} / \mathrm{año})\end{array}$ \\
\hline Zona & Norman Wells, N.W.T. & - & 1,43 \\
Artica $^{11,12}$ & Interior del Artico Canadiense & $>1$ & $1,14-3,08^{*}$ \\
\hline & Vanda & 80 & $0,87^{* *}$ \\
& Robertskollen & 120 & $0,70^{* *}$ \\
Zona & LGB00 & 186 & $0,18^{* *}$ \\
Antártica $^{13}$ & LGB10 & 390 & $0,10^{* *}$ \\
& LGB35 & 780 & $0,13^{* *}$ \\
& Vostok & 1200 & $0,05^{* *}$ \\
\hline
\end{tabular}

*La corrosión en estos lugares se midió con probetas "alambre sobre tornillo". Los valores de corrosión que aparecen en la tabla corresponden, una vez realizada la conversión (14), a probetas planas.

TABLA IV. VELOCIDADES DE CORROSIÓN DEL ACERO Y VARIABLES MEDIOAMBIENTAles Y DE CONTAMinación OBTENidas EN CuZCo, Perú.

\begin{tabular}{|c|c|c|c|c|c|c|c|c|}
\hline $\begin{array}{c}\text { Lugar de } \\
\text { exposición }\end{array}$ & $\begin{array}{c}\text { Año de } \\
\text { exposición }\end{array}$ & $\begin{array}{c}\mathrm{T} \\
{ }^{\circ} \mathrm{C}\end{array}$ & $\begin{array}{c}\mathrm{HR} \\
\%\end{array}$ & $\begin{array}{c}\mathrm{TDH} \\
\%\end{array}$ & $\begin{array}{c}\text { Precip. } \\
\mathrm{mm} / \mathrm{año}\end{array}$ & $\begin{array}{c}\mathrm{SO}_{2} \\
\mathrm{mg} / \mathrm{m}^{2} \mathrm{~d}\end{array}$ & $\begin{array}{c}\mathrm{Cl}^{-} \\
\mathrm{mg} / \mathrm{m}^{2} \mathrm{~d}\end{array}$ & $\begin{array}{c}\text { Vel. corrosión } \\
\text { acero al carbono, } \\
\mu \mathrm{m} / \text { año }\end{array}$ \\
\hline Cuzco & 1989 & 12,2 & 67 & 32,5 & 632 & Ins. & Ins. & $\begin{array}{c}1,17 \\
17\end{array}$ \\
\hline
\end{tabular}

TABla V. VELOCIDADES DE CORROSIÓN OBTENIDAS EN DIFERENTES ESTACIONES SITUADAS A UNA DISTANCIA AL MAR INFERIOR A 1 KM EN LAS ZONAS ARTICA Y ANTÁRTICA.

\begin{tabular}{|c|c|c|c|}
\cline { 2 - 4 } \multicolumn{1}{c|}{} & Lugar de exposición & $\begin{array}{c}\text { Distancia } \\
\text { al mar, } \\
\text { Km }\end{array}$ & $\begin{array}{c}\text { Velocidad de } \\
\text { corrosión del acero, } \\
(\mu \mathrm{m} / \text { año })\end{array}$ \\
\hline $\begin{array}{c}\text { Zona } \\
\text { Artica }^{12}\end{array}$ & $\begin{array}{c}\text { Zona Norte del Artico } \\
\text { Canadiense }\end{array}$ & $<1$ & $14,58-24,58^{*}$ \\
& Macquarie I. Isthmus & $<1$ & $222^{* *}$ \\
Zona & Heard I. Spit Bay & $<1$ & $55,3^{* *}$ \\
Antártica $^{13}$ & Marion I. & $<1$ & $31,9^{* *}$ \\
& Signy & $<1$ & $36,4^{* *}$ \\
& Rothera (Antarctic Pen.) & $<1$ & $27,1^{* *}$ \\
& Mawson & $<1$ & $3,35^{* *}$ \\
\hline
\end{tabular}

*La corrosión en estos lugares se midió con probetas "alambre sobre tornillo". Los valores de corrosión que aparecen en la tabla corresponden, una vez realizada la conversión (14), a probetas planas.

${ }^{*}$ Acero al carbono con la adición aproximadamente de $0,25 \%$ de cobre.

TAbla VI. Datos ANUAles (Media tRes AÑOS DE EXPOSICIÓN) DE VARIABLES MEDIOAMBIENTALES Y DE CONTAMINACIÓN, Y VELOCIDADES DE CORROSIÓN PARA EL ACERO AL CARBONO, ZINC, COBRE Y ALUMINIO, OBTENIDOS EN JUBANY (ARGENTINA) Y ARTIGAS (URUGUAY), ESTACIONES UBICADAS EN LA ANTÁRTIDA.

\begin{tabular}{|c|c|c|c|c|c|c|c|c|c|c|}
\hline \multirow{2}{*}{$\begin{array}{c}\text { Lugar de } \\
\text { exposición }\end{array}$} & \multirow{2}{*}{$\begin{array}{c}\mathrm{Cl}^{-} \\
\mathrm{mg} / \mathrm{m}^{2} \cdot \mathrm{d}\end{array}$} & \multirow{2}{*}{$\begin{array}{c}\mathrm{SO}_{2} \\
\mathrm{mg} / \mathrm{m}^{2} . \mathrm{d}\end{array}$} & \multirow{2}{*}{\begin{tabular}{c|}
$\mathrm{T}$ \\
${ }^{\circ} \mathrm{C}$
\end{tabular}} & \multirow{2}{*}{$\begin{array}{c}\mathrm{HR} \\
\%\end{array}$} & \multirow{2}{*}{$\begin{array}{c}\text { TDH } \\
\%\end{array}$} & \multirow{2}{*}{$\begin{array}{c}\text { Precip. } \\
\text { mm/año }\end{array}$} & \multicolumn{4}{|c|}{ Velocidad de corrosión } \\
\hline & & & & & & & $\begin{array}{l}\text { Acero } \\
\mu \mathrm{m} / \mathrm{a}\end{array}$ & $\begin{array}{l}\text { Zinc } \\
\mu \mathrm{m} / \mathrm{a}\end{array}$ & $\begin{array}{l}\text { Cobre } \\
\mu \mathrm{m} / \mathrm{a}\end{array}$ & $\begin{array}{c}\text { Aluminio } \\
\mathrm{g} / \mathrm{m}^{2} . \mathrm{a}\end{array}$ \\
\hline Jubany & - & Ins. & -2.7 & 84 & 29,3 & 278 & 38.1 & 1.89 & 2.00 & 4.03 \\
\hline Artigas & 159 & Ins. & $-1,7$ & 91 & 35,2 & 543 & 65,8 & - & 2,16 & 2,49 \\
\hline
\end{tabular}

\subsection{Atmósferas contaminadas por dióxido de azufre}

La estación de Svanvik, situada en Noruega, presenta una temperatura media anual de $-1,1^{\circ} \mathrm{C}$ y una cierta contaminación por dióxido de azufre $\left(13,4 \mathrm{mg} \mathrm{SO} / \mathrm{m}^{2}\right.$.d.), registrándose velocidades de corrosión de acero al carbono de 20,2 $\mu \mathrm{m} / \mathrm{a}$. Según la figura 2, a temperaturas por debajo de $0^{\circ} \mathrm{C}$ corresponderían velocidades de corrosión inferiores a la obtenida en Svanvik, por lo que parece confirmarse una vez más que la contaminación del aire atmosférico por $\mathrm{SO}_{2}$ incrementa considerablemente la velocidad de corrosión metálica, incluso a bajas temperaturas. 


\section{CONCLUSIONES}

A temperaturas extremadamente bajas (por debajo de $0^{\circ} \mathrm{C}$ ), la superficie metálica se encuentra cubierta la mayor parte del tiempo por una capa de hielo de espesor variable que limita el acceso de oxígeno y los tiempos de humectación de la superficie, disminuyendo las velocidades de corrosión hasta valores extremadamente bajos. La presencia de contaminantes atmosféricos (cloruros y dióxido de azufre) aumenta la velocidad de corrosión de los metales. Los compuestos salinos a los que dan lugar quedan retenidos en la interfase metal/hielo, acelerando el proceso de corrosión.

A temperaturas muy elevadas, bajas humedades relativas y precipitaciones muy escasas, los tiempos de humectación de la superficie metálica son muy cortos originando bajas velocidades de corrosión.

Estos valores bajos de velocidades de corrosión pueden también obtenerse en atmósferas, que aún no teniendo condiciones climáticas extremas, se caractericen por una elevada pureza del aire.

\section{BIBLIOGRAFIA}

1. ISO 9223: "Corrosion of metals and alloys. Classification of corrosivity of atmospheres", International Standards Organization (1991).

2. P.W. Brown y L.W. Masters, "Factors affecting the corrosion of metals in the atmosphere" en "Atmospheric Corrosion", Ed. W.H. Ailor, Wiley, Nueva York, 31-49 (1982).

3. S. Feliu, M. Morcillo y S. Feliu Jr., "The prediction of atmospheric corrosion from meteorological and pollution parameters.I. Annual corrosion", Corros. Sci. 34(3), 403-414 (1993).

4. M. Morcillo, B. Chico, L. Mariaca y E. Otero, "Salinity in marine atmospheric corrosion: its dependence on the wind regime existing in the site", Corros. Sci. 42, 91-104 (2000)

5. P.V. Strekalov y Yu.M. Panchenko, "The role of marine aerosols in atmospheric corrosion of metals", Prot. Met. 30, 254-263 (1994).

6. M. Morcillo, B. Chico, E. Otero y L. Mariaca, “Effect of marine aerosol on atmospheric corrosion", MP, 38(4), 72-77 (1999).

7. K. Barton, "Protection against Atmospheric Corrosion", Wiley and Sons, Londres (1976)

8. G. Schikorr, “Über die nachahmung des atmosphärischen rostens im laboratorium", Werkst. Korros., 18, 514-521 (1967).

9. M. Morcillo, E. Almeida, B. Rosales, J. Uruchurtu y M. Marrocos, “Corrosión y Protección de Metales en la Atmósferas de Iberoamérica. Parte I: Mapas de Iberoamérica de Corrosividad Atmosférica (Proyecto MICAT, XV.I/CYTED)", CYTED, Madrid (1999).

10. D. Knotkova y L. Vrobel, Proc. 11 Congreso Internacional de Corrosión, Vol. 5, AIM, Milano (1990).

11. S.K. Coburn et al., "Corrosivennes of various atmospheric test sites as measured by specimens of steel and zinc" en "Metal Corrosion in the Atmosphere", ASTM, STP 435, 360-391 (1968).

12. G.J. Biefer, "Atmospheric corrosion of steel in the Canadian Arctic" MP, January, 16-19 (1981).

13. J.D. Hughes, G.A. King y D.J. O’Brien, “Corrosivity in Antarctica - Revelations on the nature of corrosion in the world's coldest, driest, highest and purest continent", Proc. 13 Congreso Internacional de Corrosión, Melbourne, Australia (1996).

14. S.W. Dean "Comparison of corrag flat panel corrosion rates to helix corrosion rates", Report, ISO/TC156/WG4/N339

15. M. Morcillo, S. Flores, G. Salas y M. Valencia, "An extremely low corrosion rate of steel in the atmosphere of Cuzco (Perú)" Atmospheric Environment, 27A(13), 1959-1962 (1993).

16. "Climatological Normals (CLINO) for the period 1961-1990", WMO/OMM No 847, Ginebra, Suiza (1996).

17. J. A. González, “Control de la Corrosión. Estudio y Medida por Técnicas Electroquímicas", CSIC, Madrid, 297 (1996).

18. B. Rosales, A. Fernández y G. Moriena, Proc. $5^{\circ}$ Congreso Iberoamericano de Corrosión y Protección, Tenerife, España (1995).
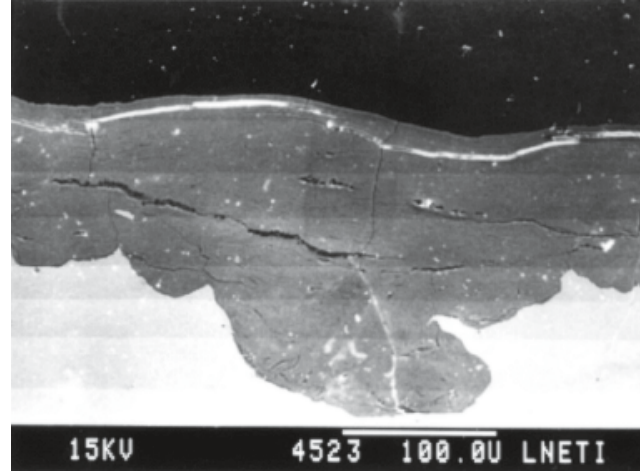

Fig. 3. Aspecto que mostraba la superficie del acero expuesto a la atmósfera marina Antártica.

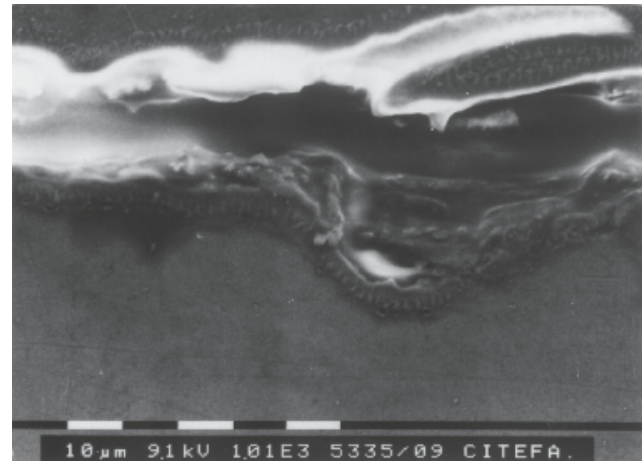

Fig. 4. Aspecto que mostraba la superficie del zinc expuesto a la atmósfera marina Antártica.

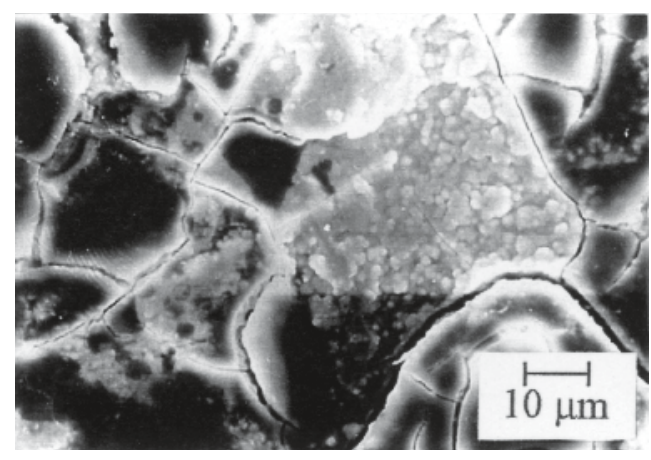

Fig. 5. Aspecto que mostraba la superficie del cobre expuesto a la atmósfera marina Antártica.

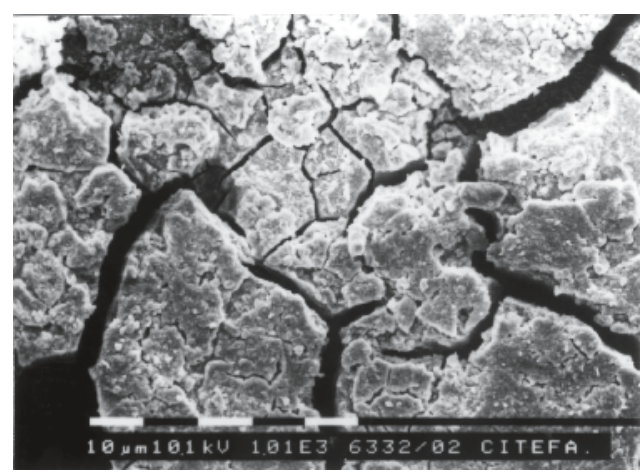

Fig. 6. Aspecto que muestra la superficie del aluminio expuesto a la atmósfera marina Antártica. 